\title{
Fat contamination of pericardial suction blood and its influence on in vitro capillary-pore flow properties in patients undergoing routine coronary artery bypass grafting
}

Micael Appelblad, ECCP

Gunnar Engström, MD, PhD

From the Heart Center, Department of Surgery and Perioperative Science, Division of Cardiothoracic Surgery, Umeå University Hospital, Umeå, Sweden.

Supported by the Swedish Society for Medical Research and funds of the Medical Faculty, Umeå University Hospital; the Swedish Medical Research Council (12X11204); the Swedish Heart and Lung Foundation, and the Heart Foundation of North Sweden.

Received for publication July 11, 2001; revisions requested Aug 21, 2001; revisions received Nov 27, 2001; accepted for publication Dec 12, 2001.

Address for reprints: K. Gunnar Engström, $\mathrm{MD}, \mathrm{PhD}$, Heart Center, Department of Surgery and Perioperative Science, Cardiothoracic Division, Umeå University Hospital, S-901 85 Umeå, Sweden (E-mail: Gunnar.Engstrom.us@vll.se).

J Thorac Cardiovasc Surg 2002;124:377-86

Copyright (C) 2002 by The American Association for Thoracic Surgery

0022-5223/2002 $\$ 35.00+0 \quad \mathbf{1 2 / 1 / 1 2 2 3 0 3}$

doi: $10.1067 / \mathrm{mtc} .2002 .122303$
Objective: Neurologic dysfunction after cardiopulmonary bypass might be due to arterial microembolization. Pericardial suction blood is a possible source of embolic material. Our aim was to determine the capillary-pore flow ability of pericardial suction blood.

Methods: Pericardial suction blood from patients undergoing coronary bypass was collected, and pericardial suction blood and venous blood were sampled at the end of cardiopulmonary bypass and before reinfusion of pericardial suction blood. Pericardial suction blood was $(\mathrm{n}=10)$ or was not $(\mathrm{n}=10)$ prefiltered through a $30-\mu \mathrm{m}$ cardiotomy screen filter before capillary in vitro analysis. Additionally, in 8 patients the plasma viscosity was measured, and in 5 of these patients, pericardial suction blood capillary deposits were evaluated by using a microscopy-imprint method and fat staining. Capillary flow was tested through $5-\mu \mathrm{m}$ pore membranes. Tested components were plasma, plasma-eliminated whole-blood resuspension, and leukocyte/plasma-eliminated erythrocyte resuspension. Initial filtration rate and clogging slope expressed the blood-to-capillary interaction.

Results: The plasma-flow profile of pericardial suction blood was highly impaired, with a $47 \%$ reduction in initial filtration rate $(P<.001)$ and a $142 \%$ steeper clogging slope flow deceleration $(P<.01)$. This difference was not due to a change in pericardial suction blood viscosity, such as by free hemoglobin, which corresponded to $5.7 \%$ of the erythrocytes. There were no differences in resuspended whole blood or erythrocytes. The cardiotomy filter had no effect. Microscopy suggested the presence of capillary fat deposits in pericardial suction blood that were not seen with venous plasma $(P<.05)$. The pericardial suction blood volume was $458 \pm 42 \mathrm{~mL}$ and contained $95.6 \pm 9.3 \mathrm{~g} / \mathrm{L}$ hemoglobin.

Conclusions: The pericardial suction blood plasma capillary flow function was highly impaired by liquid fat. Pericardial suction blood hemoglobin appears worth recovering after fat removal, despite profound hemolysis.

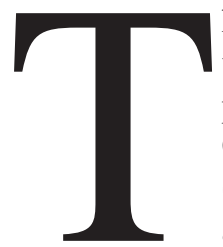

he clinical spectrum of neurologic disorders after cardiac operations varies from major stroke to diffuse symptoms detected in sensitive psychometric tests. ${ }^{1,2}$ This brain damage has several possible causes. General effects of low blood pressure and cardiopulmonary bypass (CPB) with systemic inflammatory response are plausible, but in addition, embolic mechanisms are commonly suspected. ${ }^{3}$ These embolic sources might either be air bubbles ${ }^{4}$ or particulate matter. ${ }^{5}$ They might also be described in terms of microembolism and macroembolism, which can be the result of detached particles from a calcified ascending aorta ${ }^{6}$ or plastic or silicon material generated from the CPB circuit. 7,8 These particles enter the aorta through the arterial line. Recently, the pericardial suction blood (PSB) has been suggested as a source of fat-related deposits in the brain capillaries of animals ${ }^{9}$ and human 
TABLE 1. Patient demographics

\begin{tabular}{|c|c|c|}
\hline & \multicolumn{2}{|c|}{ In vitro subgroups } \\
\hline & No cardiotomy filter & Cardiotomy filter \\
\hline \multicolumn{3}{|l|}{ Patient data } \\
\hline Sex (male/female) & $10 / 0$ & $9 / 1$ \\
\hline Age (y) & $65.3 \pm 2.7$ & $65.0 \pm 2.3$ \\
\hline Length $(\mathrm{cm})$ & $173.9 \pm 1.1$ & $175.3 \pm 2.3$ \\
\hline Weight (kg) & $76.0 \pm 2.0$ & $81.3 \pm 4.1$ \\
\hline Body surface area $\left(\mathrm{m}^{2}\right)$ & $1.91 \pm 0.03$ & $1.98 \pm 0.06$ \\
\hline Vessel disease (1-3) & $2.7 \pm 0.2$ & $2.7 \pm 0.2$ \\
\hline NYHA: I, II, IIIA, IIIB, IV (1-5) & $3.3 \pm 0.2$ & $3.5 \pm 0.2$ \\
\hline $\begin{array}{l}\text { Left ventricular function (1 } \\
\text { [good]-3 [bad]) }\end{array}$ & $1.1 \pm 0.1$ & $1.2 \pm 0.1$ \\
\hline \multicolumn{3}{|l|}{ Surgical data } \\
\hline Anastomoses (n) & $3.2 \pm 0.2$ & $3.5 \pm 0.2$ \\
\hline Surgical time (min) & $156.4 \pm 16.8$ & $157.4 \pm 8.9$ \\
\hline CPB time (min) & $61.0 \pm 5.5$ & $63.6 \pm 5.7$ \\
\hline Aortic crossclamp time (min) & $29.1 \pm 2.9$ & $32.5 \pm 2.3$ \\
\hline PSB volume (mL) & $419 \pm 53$ & $501 \pm 66$ \\
\hline Time before reinfusion (min) & $48.8 \pm 3.9$ & $57.3 \pm 4.4$ \\
\hline Surgical bleeding $(\mathrm{mL})$ & $435 \pm 121$ & $500 \pm 114$ \\
\hline Volume load (mL) & $3166 \pm 287$ & $3337 \pm 153$ \\
\hline Minimum temperature $\left({ }^{\circ} \mathrm{C}\right)$ & $34.3 \pm 0.2$ & $34.4 \pm 0.2$ \\
\hline Erythrocyte donor use (U/patient) & $0.2 \pm 0.2$ & $0.1 \pm 0.1$ \\
\hline \multicolumn{3}{|l|}{ Postoperative data } \\
\hline Length of stay (d) & $7.1 \pm 0.5$ & $8.3 \pm 0.9$ \\
\hline
\end{tabular}

Patient demographics are shown, subdivided into those in whom the PSB in vitro blood sample was or was not prefiltered through a $30-\mu \mathrm{m}$ cardiotomy filter. The New York Heart Association scale is converted to a 5-grade scale for statistical simplicity. Left ventricular function was subgrouped into 3 numeric levels. This was according to visual observations by the cardiologists on the left ventricular angiogram or preoperative echocardiography. The term surgical bleeding denotes non-PSB bleeding in the operating room. Volume load refers to the calculated difference between volume input (crystalloid and blood products) versus output (eg, urine and bleeding). The temperature was recorded in the urine bladder. Mean \pm SEM values are given, although some data are of ordinal scale.

NYHA, New York Heart Association.

subjects ${ }^{10}$ after CPB exposure. PSB is contaminated with cellular and liquid fat from the wound. ${ }^{10}$ It is further possible that activated white blood cells and platelets add to this deterioration. ${ }^{11,12} \mathrm{We}$ aimed to describe the capillary-pore flow properties of PSB with the hypothesis that fat contamination can be monitored with this technique.

\section{Materials and Methods}

\section{Patient Selection}

After institutional review board approval, patients undergoing elective, first-time, routine coronary artery bypass grafting (CABG) with CPB were enrolled successively as they appeared on the operation program. Patients were informed and provided written consent. Exclusions were ongoing anticoagulant therapy, apparent bleeding disorder, advanced peripheral vascular disease, and diabetes. In 20 patients the in vitro capillary-pore flow properties of PSB versus venous blood was measured. In 10 of these patients, selected in random sequence, the PSB was prefiltered through a $30-\mu \mathrm{m}$ cardiotomy screen filter. Patient demographics are presented in Table 1. In addition to these 20 patients, plasma viscosity of PSB versus venous blood was measured in 8 patients, and in 5 of these, the capillary membrane deposits of plasma samples were analyzed by means of microscopy.

\section{Conduct of CPB and Anesthesia}

Before $\mathrm{CPB}$, heparin $(350 \mathrm{IU} / \mathrm{kg})$ was administered to achieve an activated clotting time exceeding 480 seconds. The CPB prime solution consisted of $1400 \mathrm{~mL}$ of Ringer acetate, $60 \mathrm{~g}$ of mannitol (both from Fresenius-Kabi, Uppsala, Sweden), $160 \mathrm{mmol}$ of $\mathrm{NaCl}$, and $7500 \mathrm{IU}$ of heparin (Lövens, Ballerup, Denmark). The CPB apparatus included a membrane oxygenator with an open-circuit, integrated, venous cardiotomy, hard-shell reservoir (Quadrox, Jostra Medizintechnik, Hirrlingen, Germany) and a roller pump (Terumo-Sarns 9000 Perfusion system, Ann Arbor, Mich) set to a nonpulsatile mode, nonocclusive roller setting, with cold antegrade crystalloid cardioplegia (St Thomas Hospital II solution with added procaine hydrochloride), moderate hypothermia, acid base management after an alpha-stat regimen, and pump flow regulated to yield a venous saturation of greater than $70 \%$. Proximal coronary anastomoses were performed with a side-biting clamp. Anesthesia was maintained with intravenous fentanyl, midazolam, propofol, and pancuronium mixed with inhaled isoflurane.

\section{Collection System of PSB}

An emptied 2-L saline bag (Fresenius-Kabi) was interpositioned between the cardiotomy roller pump and the cardiotomy reservoir. The vent line was not part of the PSB system. The PSB-containing 

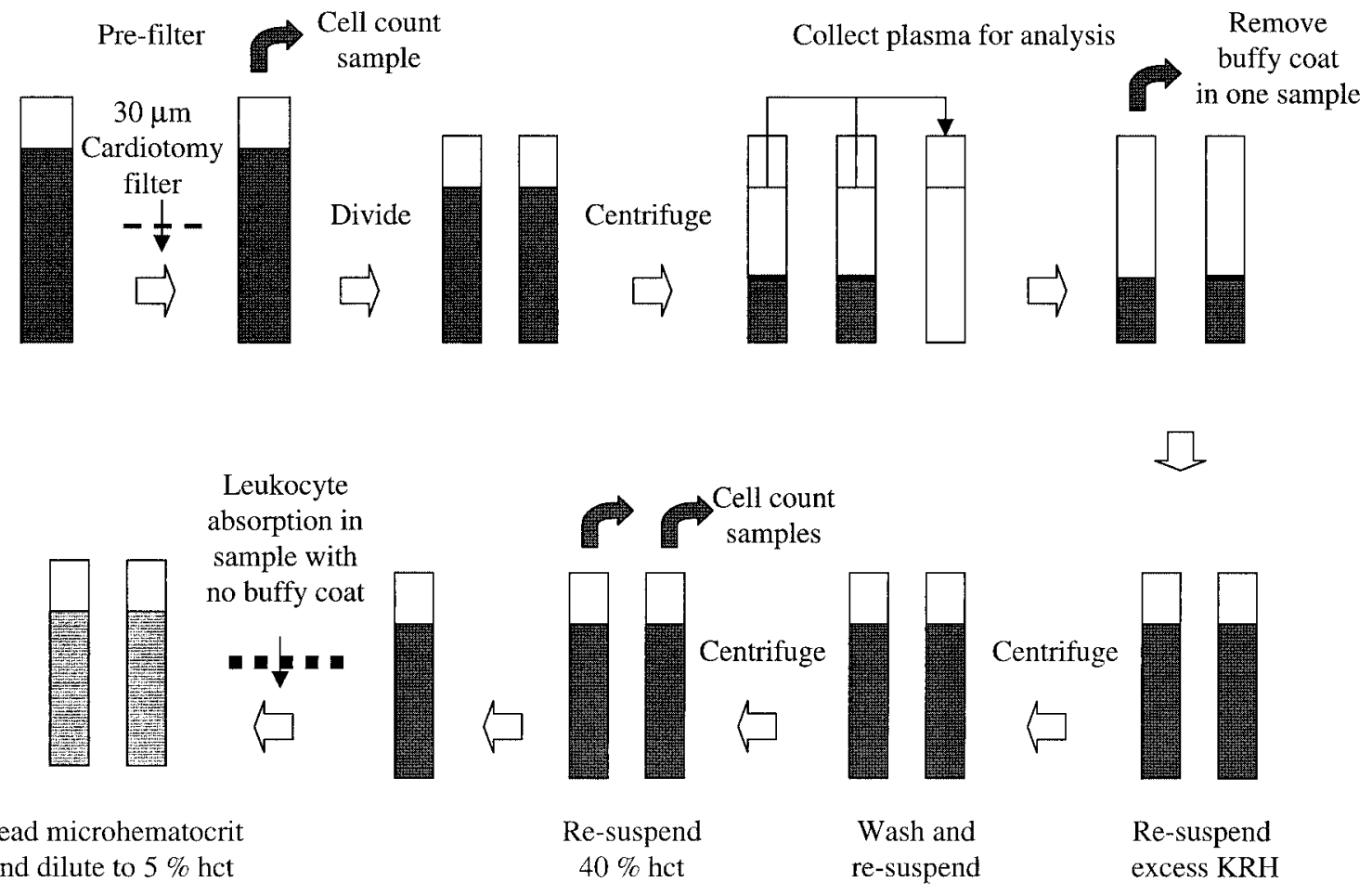

Read microhematocrit

Re-suspend

re-suspend

excess KRH

Figure 1. The blood-processing flow chart shows how blood was subdivided into plasma, plasma-eliminated whole-blood resuspension, and leukocyte/plasma-eliminated erythrocyte resuspension. See text for further details. $K R H$, Krebs-Ringer-HEPES buffer; hct, hematocrit.

bag was continuously weighed with an analog scale. The density of PSB was determined to be $1.055 \mathrm{~kg} / \mathrm{L}$, and PSB weight was converted to volume. After the release of the side-biting clamp, the collected PSB was gently mixed before blood sampling and reinfusion. Venous blood and PSB, each in 20-mL samples, were carefully aspirated from the venous reservoir inlet and from the PSB collection bag. Identical syringes were used, and the samples were sent to the laboratory for immediate analysis.

\section{Media}

A Krebs-Ringer-HEPES buffer $(\mathrm{KRH})$ was used $(\mathrm{NaCl}, 135 \mathrm{mmol} / \mathrm{L}$; $\mathrm{KCl}, 4.7 \mathrm{mmol} / \mathrm{L} ; \mathrm{KH}_{2} \mathrm{PO}_{4}, 1.2 \mathrm{mmol} / \mathrm{L}$; and N-2-hydroxyethylpiperazine-N-2-ethanesulfonic acid, $20 \mathrm{mmol} / \mathrm{L}$ ) supplemented with $0.02 \% \mathrm{wt} / \mathrm{vol}$ human serum albumin, $5.0 \mathrm{mmol} / \mathrm{L}$ glucose, and 2.56 $\mathrm{CaCl}_{2}$. The buffer was prefiltered through a $0.45-\mu \mathrm{m}$ Millipore (Millipore Corp, Bedford, Mass) membrane and had an osmolality of 300 mosm and a $\mathrm{pH}$ of 7.4 , which was calibrated by adding $\mathrm{NaOH}$.

\section{Blood Sample Processing for Capillary-Pore Analyses and Cellular Counts \\ In the initial 20 patients, PSB and venous blood were processed to explore their different subcomponents. This process is shown in Figure 1. In 10 of these samples, the PSB was allowed to drain through a cardiotomy screen filter. A cardiotomy filter was taken from inside a standard reservoir (Monolyth VRF 40; Sorin, Salug- gia, Italy) to accomplish this. This filter is a traditional nylon $30-\mu \mathrm{m}$ screen mesh from which circular pieces $(13 \mathrm{~mm})$ were}

punched out and mounted in a standard filter holder (Nuclepore Corp, Pleasanton, Calif). This prefiltering was done without applying driving force to simulate the spontaneous emptying of PSB into the cardiotomy reservoir.

From the 20-mL blood sample (venous blood and PSB), 250 $\mu \mathrm{L}$ was used for differential blood cell counts (Sysmex SE-9000; TOA Medical Electronics Co, Kobe, Japan). The remaining blood was divided into 2 equal parts that were both centrifuged for 10 minutes at $1500 \mathrm{~g}$. The plasma fraction was carefully aspirated and stored separately for capillary-pore flow analysis, and $1.5 \mathrm{~mL}$ was frozen at $-86^{\circ} \mathrm{C}$ for later analysis of free plasma hemoglobin. After plasma removal, the 2 centrifuged samples differed in that the buffy coat was either removed or allowed to remain with the erythrocytes (Figure 1). The 2 blood samples were resuspended in excess $\mathrm{KRH}$ to $50 \mathrm{~mL}$ and recentrifuged to wash out remaining plasma. This centrifugation and resuspension was repeated twice. In the sample from which the buffy coat had been aspirated, the remaining contaminating leukocyte cells were absorbed by using a leukocyte filter (Pall RC 400; Pall Biomedical Inc, Fajardo, NY). This was because buffy coat aspiration alone only removes about $50 \%$ of the leukocytes. This technique was a modified version of our previous method and is efficient in removing contaminating leukocytes and the great majority of platelets. ${ }^{13}$

After washing, the blood cells were resuspended to achieve a $40 \%$ hematocrit level. A small sample was centrifuged $(10,000 \mathrm{~g}$ for 10 minutes) for microhematocrit measurement, which was read in a microscope. On the basis of this exact cell-volume fraction, 


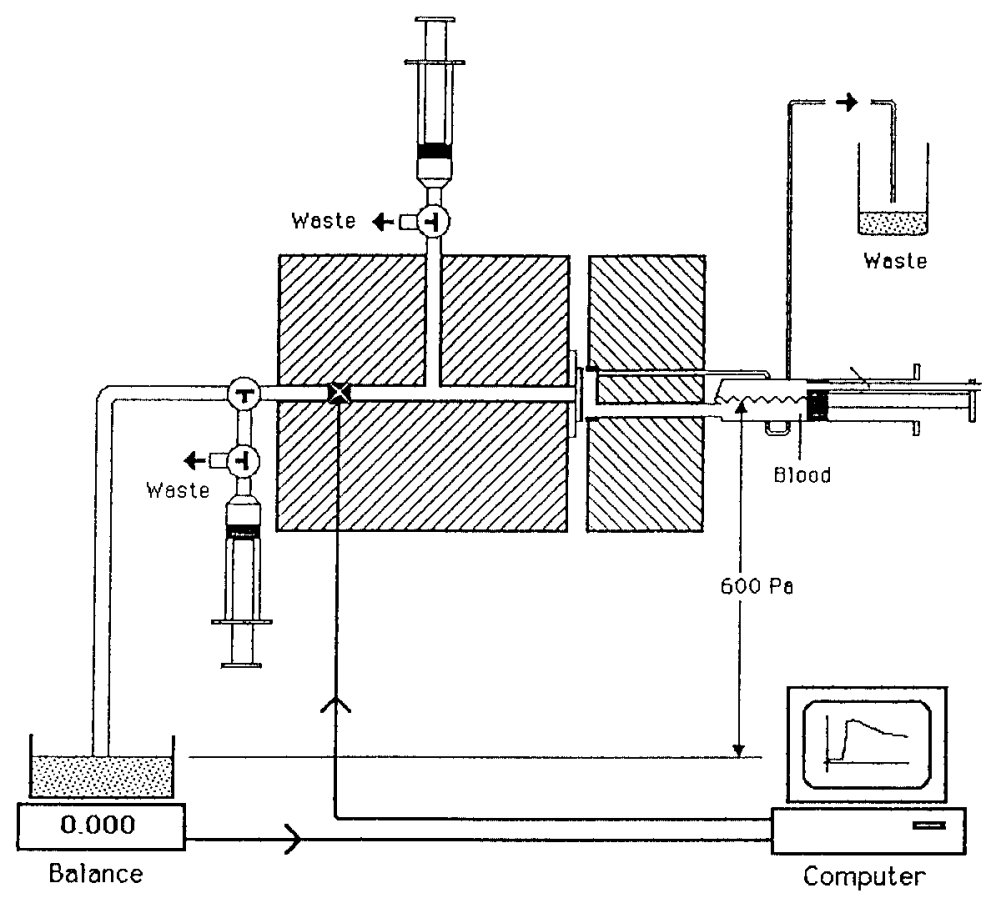

Figure 2. Computer-assisted capillary-pore flow device. The flow of medium through a capillary-pore capillary membrane is driven by constant $600-\mathrm{Pa}$ hydrostatic pressure. The onset and stop is computer controlled with a pneumatic valve and is synchronized with the data output of accumulated weight from a digital balance.

the blood was diluted to precisely reach the 5\% target hematocrit level, which is a recommended concentration for capillary-pore flow analysis. ${ }^{14}$

\section{Measurement of Capillary-Pore Flow}

The experiments were all carried out at room temperature. ${ }^{14}$ The capillary-pore flow properties of the blood subcomponents were evaluated by using polycarbonate membranes with a $5-\mu \mathrm{m}$ nominal pore diameter (pore density, $4.0 \times 10^{5}$; SN: 113613 Lot: 54M8B46; Nuclepore Corp, Pleasanton, Calif). The filters were mounted in the KGE flowmeter (ESTRAB, Umeå, Sweden; Figure 2), which has been well described elsewhere. ${ }^{15} \mathrm{~A}$ hydrostatic constant driving pressure of $600 \mathrm{~Pa}$ was used. A digital balance (Mettler PM 480 Deltarange; Mettler instrumente AG, Greifensee, Switzerland) measurement was continuously acquired by using a standard personal computer. The onset and termination ( 3.15 seconds) of the flow was controlled by the computer through a pneumatic slide valve. The flow was derived from an accumulated volume at a $7.5-\mathrm{Hz}$ sampling rate, assuming a $1.0 \mathrm{~kg} / \mathrm{L}$ density of the low hematocrit resuspension (approximately $1 \mathrm{mg}-1 \mu \mathrm{L}$ ). Each filter was calibrated with a blank run before each blood sample (see flow curve in Figure 3, upper panel). At flow onset, there is acceleration before a steady state is reached. The acceleration phase was omitted from the calculations. ${ }^{15}$ All presented data refer to the 1 - to 3 -second window. At steady state, the flow declines with time as a result of a gradual occlusion of the pores by particles or rigid cells. The negative curve slope is an indicator for capillary-occluding property. The results are presented as relative flow (blood sample divided by blank medium; Figure 3, lower vs upper panel) to compensate for filter variations. From a linear regression of the relative curve, 1 to 3 seconds, the intercept at 1 second expresses the initial filtration rate (IFR). The decline in relative flow represents the clogging slope (CS). It is important to emphasize that as plasma was removed and the blood cells were resuspended in $\mathrm{KRH}$, protein-induced cell aggregation did not occur in the experiments. The platelets are present in the plasma sample but are reduced in number to about $30 \%$ in the whole-blood resuspension or near completely eliminated in the erythrocyte resuspension because they are trapped in the leukocyte prefilter.

\section{Measurements of Viscosity, Free Hemoglobin, and Microscopy of Capillary Filter Deposits}

The blood was sampled as described above, including through a $30-\mu \mathrm{m}$ cardiotomy screen. The 2 samples were centrifuged ( $1500 \mathrm{~g}$ for 10 minutes), and the plasma fraction was carefully removed for analysis. Although IFR can be regarded as a relative viscosity measurement (multichannel capillary-pore viscometer), these data are difficult to interpret because of cell/particle-pore interaction. The plasma viscosity was therefore measured with a standard capillary tube viscometer (KIMAX-46460-50; Cannon Instrument Co, State College, Pa). With this device, the time was recorded during a 3.5-mL plasma flow through a $50-\mu \mathrm{m}$ diameter and a 75-mm-long capillary under an average driving pressure of $600 \mathrm{~Pa}$. This was performed at room temperature to mimic the capillary-pore flow results. The flow time of plasma versus water provided the 
relative viscosity. The plasma-free hemoglobin was measured by means of spectrophotometry (DU 640; Beckman Coulter, Inc, Fullerton, Calif). The absorbance was measured at 400 and $430 \mathrm{~nm}$ (baseline mean) versus $415 \mathrm{~nm}$ (hemoglobin). A calibration curve was developed with a blood sample of known hemoglobin concentration, from which precise and complete lysis at different concentrations was created by means of resuspension in water.

A microscopy method was developed with the aim of demonstrating the mechanisms behind differences in plasma capillarypore flow. The 5- $\mu \mathrm{m}$ Nuclepore membrane was mounted in a 13-mm Pop-Top filter holder (Nuclepore Corp), through which 1 $\mathrm{mL}$ of plasma was filtered. The membrane deposit was imprinted on a noncoated microscope glass slide. The imprint was air-dried for 30 seconds and fixed in $10 \%$ formaldehyde in phosphate buffer, followed by staining without dehydration. The staining had a hydrophilic-basophilic component (Hematoxylin-Mayer) and a hydrophobic fat stain (oil red in 50\% isopropanol). After drying, a cover slip was mounted with gelatin. The occurrence and type of deposit was evaluated under the microscope. Because the great majority of deposits consisted of fat-positive material, a $10 \times$ objective (Carl Zeiss, Obekochen, Germany) was used to provide an optimal overview. The slides were coded, and the amounts of deposits were evaluated with a semiquantitative scale (minimummaximum $=0-4)$. The observer was blinded to the slide identity.

\section{Statistics}

Analysis for differences between venous blood and PSB was done with the paired Student $t$ test. Differences between groups of patients, with and without a cardiotomy filter, were determined by means of unpaired testing. The results demonstrated a close to normal distribution. The capillary-pore flow data were evaluated by using linear regressions with a least-sum square method. The microscopy data were evaluated by means of exact probability calculation. Mean \pm SEM values are given.

\section{Results}

\section{Clinical Results}

The volume of collected PSB was, on average, $458 \pm 42$ $\mathrm{mL}$ (range, 250-1000 mL). No donor blood was needed during CPB (surgical data are shown in Table 1).

\section{Blood Cell Characteristics, PSB Versus Venous Blood}

The PSB differed from venous blood (Table 2). The 30- $\mathrm{mm}$ cardiotomy filter did not affect the PSB cell counts, and in Table 2 the results were pooled $(n=20)$. The PSB and venous blood had similar hemoglobin concentrations, and there were no artifacts from hemodilution. However, the leukocyte counts showed differences between groups. The overall numbers were reduced in PSB, which solely reflects the neutrophilic cells. Platelets were found to be increased compared with levels seen in venous blood. For comparison, the blood cell counts before and after the operation are also shown (Table 2).
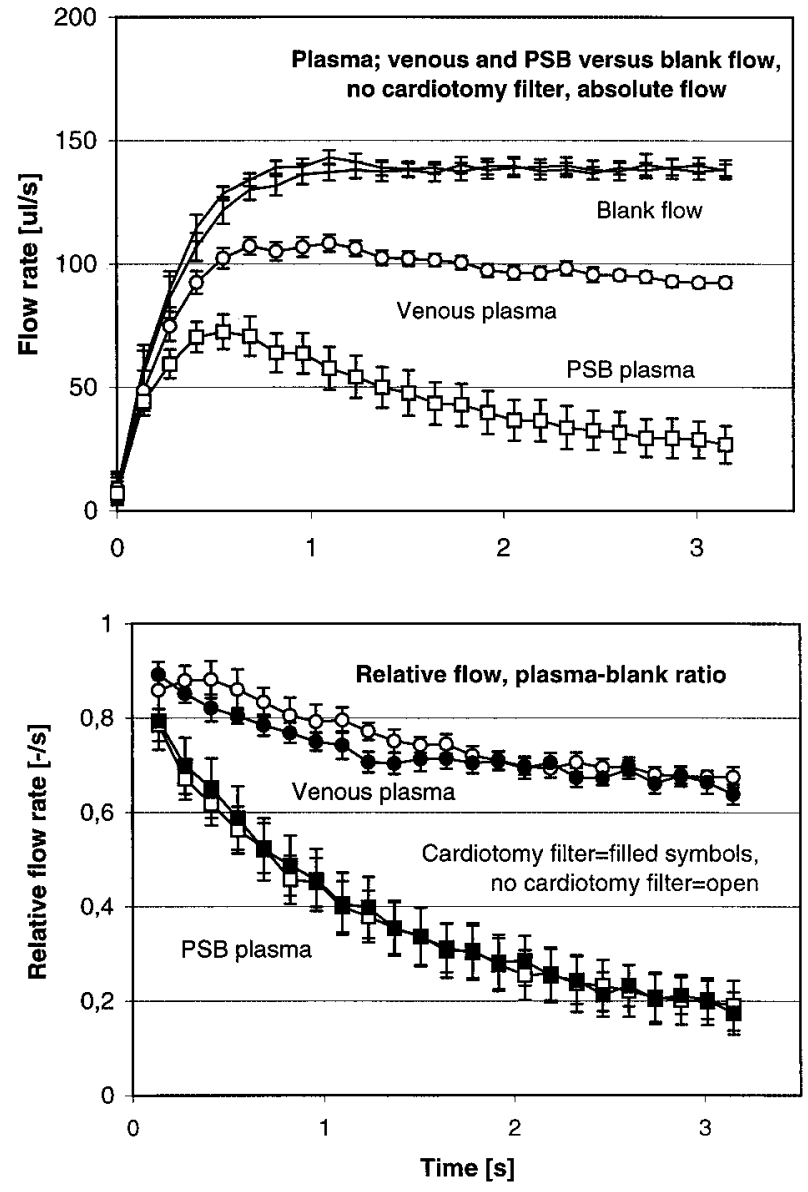

Figure 3. Flow curves of PSB and venous blood plasma. Upper panel, The absolute flow rate versus time shows an obvious acceleration that ends at about 1 second for blank medium. This period is omitted from the calculations, and all presented data refer to the measuring window of 1 to 3 seconds. The 2 curves at the top show the flow curve of buffer (blank flow), whereas that for venous plasma (open circles) and PSB plasma (open squares) demonstrate a depressed flow rate. Lower panel, Each sample is tested versus the blank medium to calculate the relative flow rate, which is indicated. This is the inverse of relative viscosity, although this model is more sensitive to suspended particles and cells. Open and filled circles indicate relative flow of venous plasma in the 2 study groups without and with 30- $\mu \mathrm{m}$ cardiotomy prefiltering, respectively. Open and filled squares indicate relative flow of unfiltered PSB plasma and $30-\mu \mathrm{m}$ prefiltered PSB plasma, respectively. There are 10 observations in each group. Data points denote mean values \pm SEM.

\section{Capillary-Pore Flow Properties of PSB Versus Venous Plasma}

The PSB plasma-flow profile was highly impaired, both in terms of IFR and CS (Figure 3, upper panel). The flow data are better expressed on their relative scale versus blank flow to compensate for filter variations (Figure 3, lower panel; Table 3 ). The addition of the $30-\mu \mathrm{m}$ cardiotomy filter did 
TABLE 2. Differences between venous blood and PSB

\begin{tabular}{|c|c|c|c|c|}
\hline Pooled $(n=20)$ paired statistics & Venous, before operation & Venous, during CPB & PSB, during CPB & Venous, day 1 ICU \\
\hline Leukocyte count $\left(\times 10^{9} / \mathrm{L}\right)$ & $5.77 \pm 0.32$ & $8.08 \pm 0.66^{* *}$ & $5.88 \pm 0.60 \dagger \dagger$ & $9.57 \pm 0.45^{* * *}, \ddagger$ \\
\hline Neutrophilic cells $\left(\times 10^{9} / \mathrm{L}\right)$ & & $5.60 \pm 0.59$ & $2.84 \pm 0.51 \dagger \dagger \dagger$ & \\
\hline Eosinophilic cells $\left(\times 10^{9} / \mathrm{L}\right)$ & & $0.14 \pm 0.02$ & $0.57 \pm 0.08 \mathrm{t \dagger \dagger}$ & \\
\hline Basophilic cells $\left(\times 10^{9} / \mathrm{L}\right)$ & & $0.03 \pm 0.01$ & $0.11 \pm 0.01 \dagger \dagger$ & \\
\hline Lymphocytes $\left(\times 10^{9} / \mathrm{L}\right)$ & & $2.10 \pm 0.14$ & $2.12 \pm 0.24$ & \\
\hline Monocytes $\left(\times 10^{9} / \mathrm{L}\right)$ & & $0.21 \pm 0.03$ & $0.23 \pm 0.03$ & \\
\hline Erythrocyte count $\left(\times 10^{12} / \mathrm{L}\right)$ & $4.95 \pm 0.08$ & $3.46 \pm 0.17^{* * *}$ & $3.17 \pm 0.30$ & $3.80 \pm 0.09^{* * *}$ \\
\hline Hemoglobin (g/L) & $145.8 \pm 2.0$ & $97.6 \pm 7.0^{* * *}$ & $95.6 \pm 9.3$ & $112.4 \pm 2.6^{* * *}$ \\
\hline Hematocrit $(\%)$ & $44.2 \pm 0.6$ & $30.5 \pm 1.6^{* * *}$ & $28.1 \pm 2.7$ & $34.3 \pm 0.8^{* * *}$ \\
\hline Mean cell volume (fL) & $89.3 \pm 0.6$ & $88.3 \pm 0.6^{* * *}$ & $88.7 \pm 0.6 \dagger$ & $90.3 \pm 0.7^{* *}$, \\
\hline Platelet count $\left(\times 10^{9} / \mathrm{L}\right)$ & $206.5 \pm 8.3$ & $76.9 \pm 7.8^{* * *}$ & $104.4 \pm 7.2 \dagger \dagger$ & $156.3 \pm 7.8^{* * *}$, \\
\hline
\end{tabular}

Cellular counts and concentrations before, during, and after surgical intervention. Both venous blood and PSB are shown during CPB with leukocyte differential counts. The patients were pooled $(n=10+10)$. Mean \pm SEM values are given.

Statistical differences are shown with the following symbols: * denotes difference versus preoperative blood, $\uparrow$ denotes PSB versus venous blood during $\mathrm{CPB}$, and $\ddagger$ denotes venous blood postoperatively versus venous blood during CPB (single symbol, $P<.05$; double symbol, $P<.01$, triple symbol, $P<.001$ ). ICU, Intensive care unit.

not improve the PSB plasma-flow properties. The IFR at 1 second (Table 3) illustrates the relative effect of plasma in this model. The plasma-flow properties did not correlate with plasma platelet counts.

\section{Flow Properties of Resuspended Whole-Blood Cells (Leukocytes Remaining)}

With 5\% washed whole-blood cells in suspension, the flow decreased versus that of plasma (Table 3). There were no detectable differences between the behavior of venous blood and PSB (Figure 4). In this setting the leukocytes have a great effect on the filter-occluding properties, and leukocyte counts showed a strong inverse association to flow. The IFR was sensitive to neutrophilic and macrophage counts $(P<.05$ and $P<.01$ for linear correlation, respectively). The CS was sensitive to lymphocyte and platelet counts $(P<.05)$. The addition of the $30-\mu \mathrm{m}$ cardiotomy filter appeared to decrease clogging, as indicated by the CS measurement $(P<.05$, Table 3$)$.

\section{Flow Properties of Resuspended Erythrocytes}

When the buffy coat and remaining leukocytes were removed, the flow of 5\% erythrocytes was improved versus that with the buffy coat remaining. This difference expresses the relative magnitude of leukocyte contribution to the results, and the CS was reduced $(P<.05$ and $P<.01$, Figures 4 and 5, respectively). PSB did not differ from that of venous blood, and the $30-\mu \mathrm{m}$ filter did not add detectable changes (Figure 5). However, when the 2 groups were pooled $(\mathrm{n}=20)$, there was a close-to-significant $(P<.06)$ steeper CS in PSB, possibly indicating an erythrocyte trauma.

\section{Plasma-Free Hemoglobin and Viscometry}

The PSB had macroscopic hemolysis, and the free hemoglobin was $5478 \pm 479 \mathrm{mg} / \mathrm{L}$ compared with $48 \pm 7 \mathrm{mg} / \mathrm{L}$ in venous blood $(P<.001)$. The contribution of PSB to the venous free hemoglobin on reinfusion was calculated, assuming a fixed plasma distribution volume of $5 \mathrm{~L}$ and an additional $1.4 \mathrm{~L}$ of priming. The PSB and venous hematocrit and free hemoglobin levels allowed calculation of an expected free hemoglobin level of $455 \mathrm{mg} / \mathrm{L}$. The PSB therefore accounts for about $89 \%$ of the measured free hemoglobin at the end of CPB.

The PSB free hemoglobin demonstrated no correlation to PSB plasma viscosity. The plasma relative viscosity was not different between PSB and venous plasma (1.35 \pm 0.07 vs $1.33 \pm 0.06$, respectively).

\section{Microscopy Results}

The filter imprint revealed deposits of fat-positive staining in PSB. In the microscopic evaluation the PSB showed positive results in 5 of 5 experiments $(P<.05)$. In terms of the semiquantitative scale ( $0-4$ points), the venous plasma showed an average result of 0.6, and PSB plasma showed and average result of 3.2 (median, 1 vs 3 ). Typical deposits are demonstrated in Figure 6.

\section{Discussion}

An undesired outcome of routine cardiac operations is brain damage, and various risk factors have been identified. ${ }^{16}$ Despite extensive research, this problem remains without a definitive solution. ${ }^{1,2,17} \mathrm{~A}$ recent concern is the recycling of PSB. PSB might contribute to the systemic inflammatory response that occurs after $\mathrm{CPB} .^{3}$ It is known today that PSB also contains fat from the wound. ${ }^{10}$ Liquid fat is not a natural part of blood, which might be exemplified by fatresembling microemboli (small capillary and arteriolar dilations) found in the small-diameter arteries of the brain after CPB. ${ }^{9,10}$

We have previously studied the capillary-pore flow func- 
TABLE 3. Capillary-pore flow data

\begin{tabular}{|c|c|c|c|c|}
\hline & \multicolumn{4}{|c|}{ In vitro blood sample processing } \\
\hline & \multicolumn{2}{|c|}{ No cardiotomy filter } & \multicolumn{2}{|c|}{ Cardiotomy filter } \\
\hline & Venous & PSB & Venous & PSB \\
\hline \multicolumn{5}{|l|}{ Plasma } \\
\hline IFR & $0.777 \pm 0.022$ & $0.398 \pm 0.056^{* * *}$ & $0.735 \pm 0.021$ & $0.407 \pm 0.066^{* * *}$ \\
\hline $\operatorname{CS}\left(s^{-1}\right)$ & $-0.0551 \pm 0.0121$ & $-0.1096 \pm 0.0090^{* *}$ & $-0.0376 \pm 0.0057$ & $-0.1146 \pm 0.0144^{* * *}$ \\
\hline \multicolumn{5}{|c|}{ Resuspended with leukocytes } \\
\hline IFR & $0.550 \pm 0.032$ & $0.514 \pm 0.039$ & $0.459 \pm 0.038$ & $0.498 \pm 0.048$ \\
\hline $\operatorname{CS}\left(s^{-1}\right)$ & $-0.1048 \pm 0.0108$ & $-0.1155 \pm 0.0109$ & $-0.0924 \pm 0.0085$ & $-0.0806 \pm 0.0105$ \\
\hline \multicolumn{5}{|c|}{ Resuspended erythrocytes } \\
\hline IFR & $0.582 \pm 0.034$ & $0.567 \pm 0.032$ & $0.578 \pm 0.048$ & $0.545 \pm 0.048$ \\
\hline $\operatorname{CS}\left(s^{-1}\right)$ & $-0.0644 \pm 0.0122$ & $-0.0763 \pm 0.0106$ & $-0.0435 \pm 0.0105$ & $-0.0655 \pm 0.0105$ \\
\hline
\end{tabular}

Capillary-pore flow data, IFR and CS are shown for PSB versus venous blood subdivided into plasma, plasma-eliminated whole-blood resuspension, and leukocyte/plasma-eliminated erythrocyte resuspension groups. The patients were grouped to test the in vitro effects of a 30- $\mu \mathrm{m}$ cardiotomy filter versus without a filter. Mean \pm SEM values are given ( $n=10$ in each group). Statistical differences are shown between PSB and venous blood in each group $\left.{ }^{* *} P<.01 ;{ }^{* *} P<.001\right)$. Additional statistical results are described in the text.

tion of autotransfusion blood and fat contaminants after knee operations ${ }^{18,19}$ and found markedly impaired results in terms of plasma flow. This finding was interpreted as being caused, in large part, by contaminating particles. After cardiac operations, mediastinal drain blood is autotransfused into the venous system, and clogging material might end up in the pulmonary circulation. However, during cardiac operations and $\mathrm{CPB}$ arterial reinfusion, such debris can be expelled into the cerebral circulation. Furthermore, in a previous study of ours ${ }^{20}$ on CPB-induced blood changes, it was found that leukocytes triggered a marked reduction in $5-\mu \mathrm{m}$ capillary-pore flow. This appeared both as leukocyte activation but also as a result of leukocytosis during CPB. Of great interest was the finding that the plasma improved in capillary-pore flow behavior because of the crystalloid prime at $\mathrm{CPB}$ onset but was then significantly reduced during CPB. This suggested a mechanism of gradual particle contamination from the continuously added PSB into the CPB circuit.

This study is a follow-up to our previous work ${ }^{20}$ and was designed to describe the capillary-pore flow properties of PSB in an in vitro model. For this purpose, PSB was tested in a specially designed PSB collection system. From a clinical point of view, this system worked without apparent effects on CPB management or patient safety. The patients tolerated this retained volume. The PSB was poor in leukocytes, mainly neutrophilic cells. The hemoglobin concentration was equal to that of venous blood. The mechanisms behind the neutropenia in the PSB are unknown but can in theory reflect an activation and adhesion to the wound tissue.

We used a method to measure the flow of controlled suspensions of blood through well-defined narrow capillary pores, here of $5 \mu \mathrm{m}$ in diameter and $11 \mu \mathrm{m}$ in length. These pores and applied methods are well described in the literature ${ }^{14}$ and have many clinical implications. ${ }^{21}$ However, over the years, artifacts have been described, including by our group. ${ }^{15}$ Although the experimental principle might appear simple, the biophysics are complex. However, the method used in this study provided results that might help in the understanding of how blood works in human capillaries during CPB and PSB reinfusion. Our initial focus was on PSB leukocytes, and the method again proved sensitive to leukocytes. However, the leukocyte contribution to the positive findings was negligible in comparison with PSB plasma characteristics. When PSB plasma was compared with that of venous plasma, an obvious deterioration was detected. The PSB plasma capillary flow indicated a markedly increased capillary clogging ability, and at 3 seconds, the flow was only about $30 \%$ of that for venous plasma and, in fact, was close to zero in many of the experiments. The leukocyte flow spectrum was studied by using resuspended whole-blood cells, but PSB did not differ from venous blood in this respect. The erythrocytes were tested after leukocyte absorption but were also unaffected. One reservation was a close-to-significant steeper CS slope in PSB erythrocytes, which suggests cellular trauma. PSB demonstrated major hemolysis, but the nonlysed cells functioned well. It is important to emphasize that the blood cells were washed and free from plasma, and therefore cell aggregation did not occur. The PSB hemolysis constituted a major portion of free hemoglobin detected in the circulation, as described by others. ${ }^{22}$ Another concern that is often recognized is the effect of platelet activity in CPB systems. The whole-blood fraction of resuspended cells correlated with not only leukocytes (CS) but also with platelet counts (IFR). Platelets were found in the plasma fraction, but plasma-flow properties did not correlate with platelet counts. In general, 

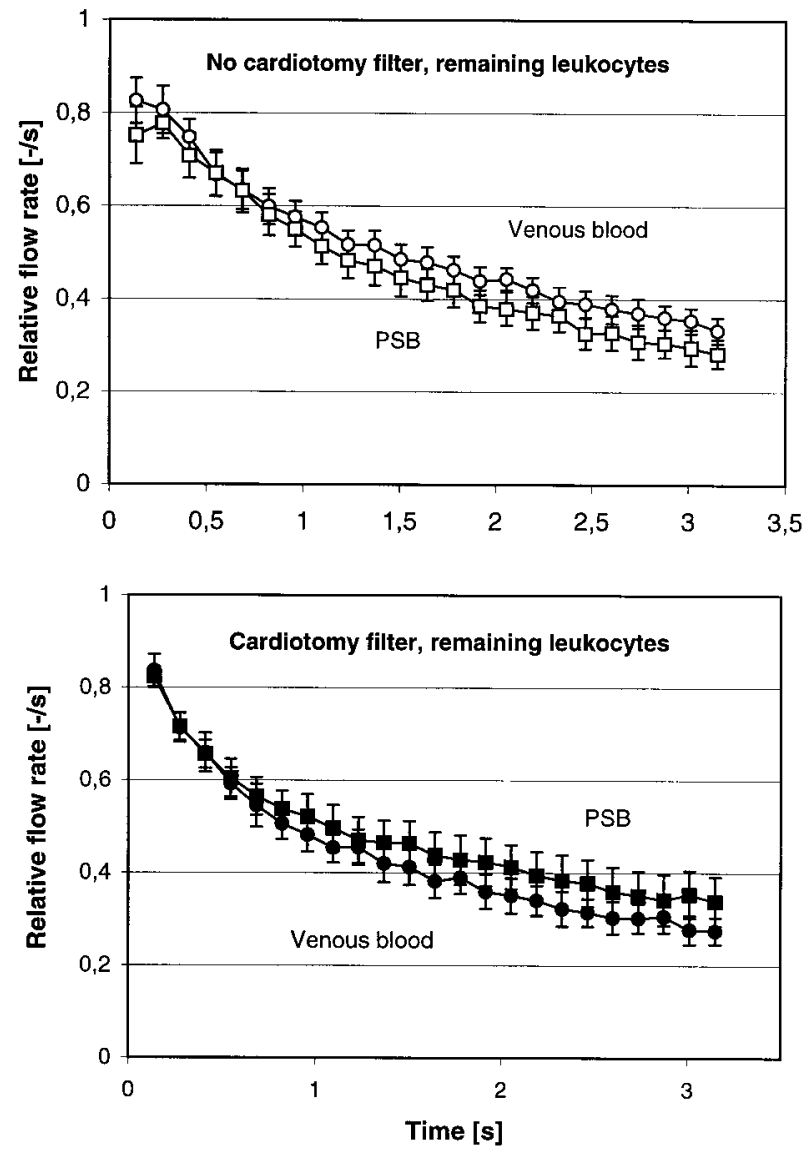

Figure 4. The relative flow curves of PSB and venous whole-blood resuspensions with remaining leukocytes. Upper panel, Open circles and squares indicate flow results without $30-\mu \mathrm{m}$ cardiotomy prefiltering of venous and PSB blood cells, respectively. Lower panel, Filled circles and squares indicate flow results with $30-\mu \mathrm{m}$ cardiotomy prefiltering of venous and PSB blood cells, respectively. There are $\mathbf{1 0}$ observations in each group. Data points denote mean values \pm SEM.

platelets have a smaller diameter to that of the in vitro capillaries used in this model and therefore do not interfere with the results. The effect of a 30- $\mu$ m cardiotomy filter was also tested, but there was no evidence of beneficial function in this respect.

The impaired PSB plasma flow can be explained by 2 possible mechanisms: either the viscosity is changed or the plasma contained contaminating particles. The PSB viscosity was tested but did not demonstrate differences versus the venous plasma. Contaminating particles were investigated by means of a newly developed microscopy method. Filter deposits were imprinted on to a glass slide for fixation and staining. Two stains were added to examine the possibility of either basophilic cellular presence, such as platelets and
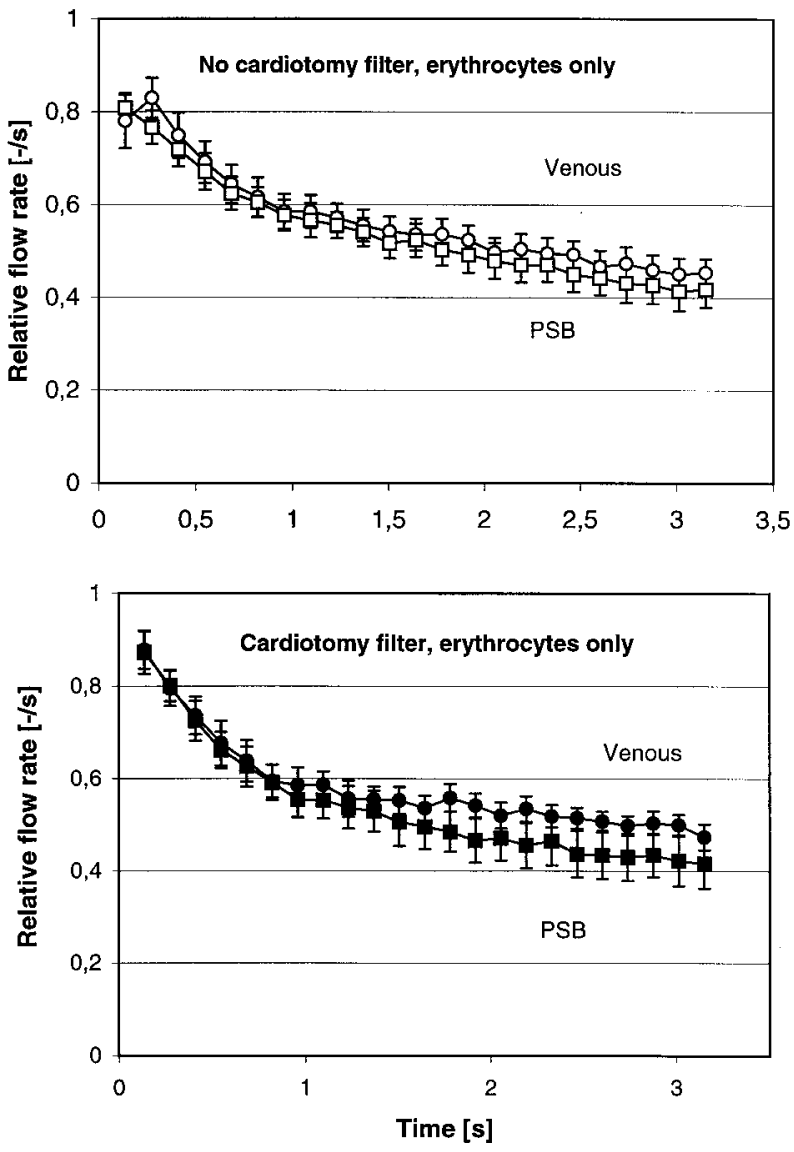

Figure 5. The relative flow curves of PSB and venous erythrocyte resuspensions with eliminated leukocytes. Upper panel, Open circles and squares indicate flow results without $30-\mu \mathrm{m}$ cardiotomy prefiltering of venous and PSB erythrocytes, respectively. Lower panel, Filled circles and squares indicate flow results with $30-\mu \mathrm{m}$ cardiotomy prefiltering of venous and PSB erythrocytes, respectively. There are 10 observations in each group. Data points denote mean values \pm SEM.

leukocytes, or hydrophobic material, such as fat. The presence of fat was clear (Figure 6).

In conclusion, these results support previous findings that suggested the presence of fat microemboli of PSB..$^{10}$ This method might help in the development of new means to monitor and avoid fat contamination from PSB during CPB. Although it remains difficult to extrapolate the in vitro findings to clinical consequences, these findings are of enough magnitude to strongly question the routine use of unprocessed PSB.

We thank Mrs Erica Jansson, perfusionist; Mrs Berith Lundström, laboratory technician, for blood sampling and laboratory assistance; and Dr Michael Haney for language editing. 

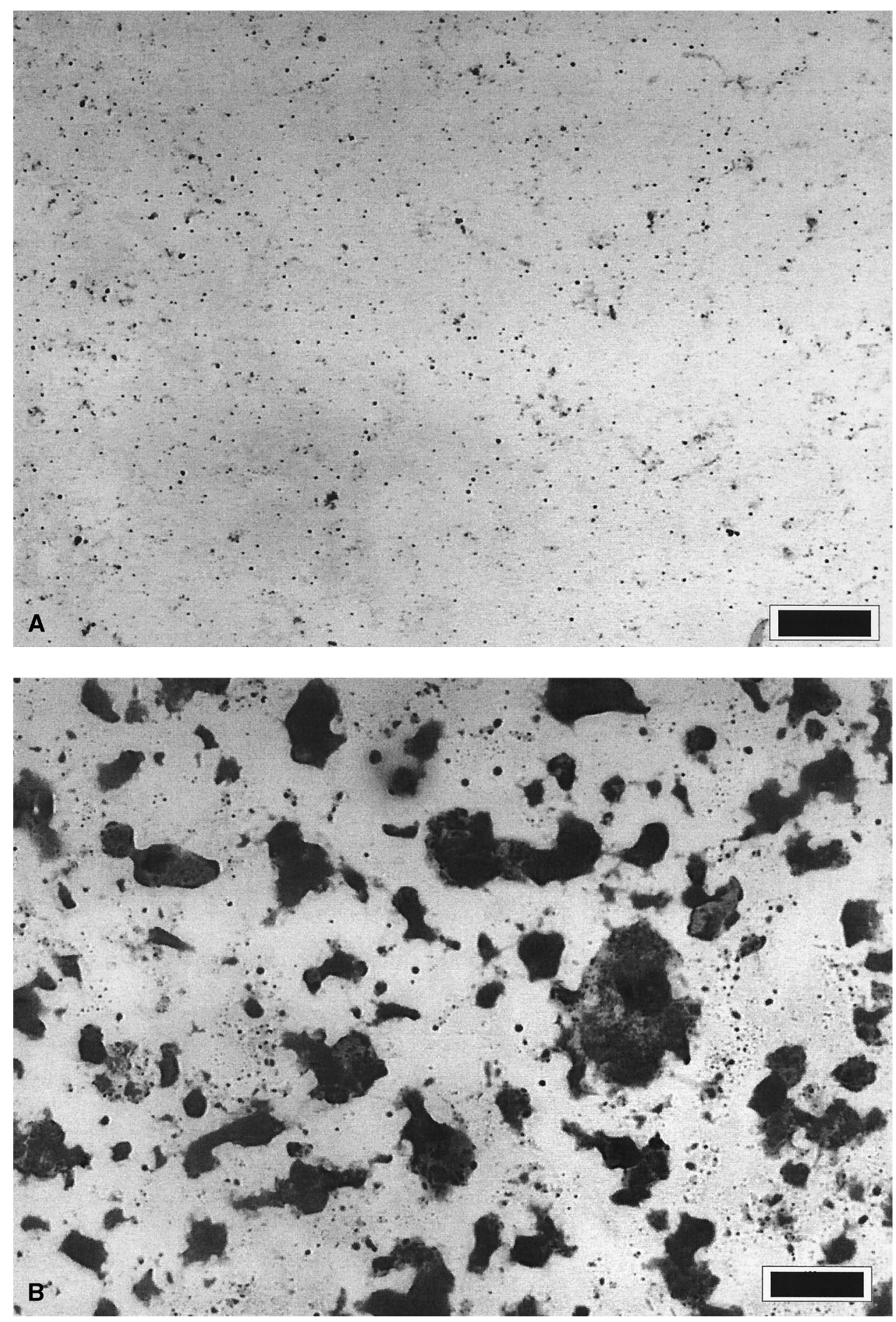

Figure 6. A typical result from the 5- $\mu \mathrm{m}$ filter imprint method: upper panel, venous plasma; lower panel, PSB plasma. A scale bar is depicted at the bottom right of each panel to indicate $100 \mu \mathrm{m}$. This PSB-to-venous difference was seen consistently in $\mathbf{5}$ of $\mathbf{5}$ test results. 


\section{References}

1. Hammon JW Jr, Stump DA, Kon ND, Cordell AR, Hudspeth AS, Oaks TE, et al. Risk factors and solutions for the development of neurobehavioral changes after coronary artery bypass grafting. Ann Thorac Surg. 1997;63:1613-8.

2. Stump DA, Jones TJ, Rorie KD. Neurophysiologic monitoring and outcomes in cardiovascular surgery. J Cardiothorac Vasc Anesth. 1999;13:600-13.

3. Taylor KM. Brain damage during cardiopulmonary bypass. Ann Thorac Surg. 1998;65:20-6.

4. Kurusz M, Butler B, Katz J, Conti VR. Air embolism during cardiopulmonary bypass. Perfusion. 1995;10:361-91.

5. Barbut D, Hinton RB, Szatrowski TP, Hartman GS, Bruefach M, Williams-Russo $\mathrm{P}$, et al. Cerebral emboli detected during bypass surgery are associated with clamp removal. Stroke. 1994;25:2398-402.

6. Stump DA, Rogers AT, Hammon JW, Newman SP, Phil D. Cerebral emboli and cognitive outcome after cardiac surgery. J Cardiothorac Vasc Anesth. 1996;10:113-9.

7. Moody DM, Brown WR, Challa VR, Stump DA, Reboussin DM, Legault, et al. Microemboli associated with cardiopulmonary bypass: a histologic and magnetic resonance imaging study. Ann Thorac Surg. 1995;59:1304-7

8. Challa VR, Lovell MA, Moody DM, Brown WR, Reboussin DM, Markesbery WR. Laser microprobe mass spectrometric study of aluminum and silicon in brain emboli related to cardiac surgery. $\mathrm{J} \mathrm{Neu}$ ropathol Exp Neurol. 1998;57:140-7.

9. Kincaid EH, Jones TJ, Stump DA, Brown WR, Moody DM, Deal DD, et al. Processing scavenged blood with a cell saver reduces cerebral lipid microembolization. Ann Thorac Surg. 2000;70:1296-300.

10. Brown WR, Moody DM, Challa VR. Cerebral fat embolism from cardiopulmonary bypass. J Neuropathol Exp Neurol. 1999;58:109-19.

11. Gu YJ, van-Oeveren W, Boonstra PW, de-Haan J, Wildevuur C Leukocyte activation with increased expression of CR3 receptors during cardiopulmonary bypass. Ann Thorac Surg. 1992;53:83943.

12. Utley JR. Cardiopulmonary bypass surgery. Curr Opin Cardiol. 1992; 7:267-75.

13. Lindmark K, Engström KG. Use of polyester leukocyte elimination filters in blood filterability research. Biorheology. 1998;35:131-40.

14. Bull BS, Chien S, Dormandy JA, Kieswetter H, Lewis SM, Lowe GDO, et al. Guidelines for measurement of blood viscosity and erythrocyte deformability. Clin Hemorheol. 1986;6:439-53.

15. Lindmark K, Engström KG. Analysis of flow acceleration during erythrocyte filtration: dependence of hematocrit and cell rigidity. Biorheology. 1996;33:379-95.

16. Hougue CW, Murphy SF, Schechtman KB, Dávila-Román VG. Risk factor for early or delayed stroke after cardiac surgery. Circulation. 1999;100:642-47.

17. Åberg T, Kihlgren M. Cerebral protection during open-heart surgery. Thorax. 1977;32:525-33.

18. Dalén T, Engström KG. Filterability of autotransfusion blood cells and plasma after total knee arthroplasty. Clin Hemorheol Microcirc. 1998; 19:181-95

19. Dalén T, Engström KG. Microrheology of filtered autotransfusion drain blood with and without leukocyte reduction. Clin Hemorheol Microcirc. 1999;21:113-23.

20. Lindmark K, Engström KG. Effects of cardiopulmonary bypass on narrow-pore flow behavior of blood. J Thorac Cardiovasc Surg. 2002; 123:381-3.

21. Mokken FC, Kedaria M, Henny CP, Hardeman MR, Gelb AW. The clinical importance of erythrocyte deformability, a hemorheological parameter. Ann Hematol. 1992;64:113-22.

22. Hansbro SD, Sharpe DAC, Catchpole R, Welsh KR, Munsch CM, McGoldrick JP, et al. Haemolysis during cardiopulmonary bypass: and in vivo comparison of standard roller pumps, nonocclusive roller pumps and centrifugal pumps. Perfusion. 1999;14:3-10.

\section{Availability of Journal back issues}

As a service to our subscribers, copies of back issues of The Journal of Thoracic and Cardiovascular Surgery for the preceding 5 years are maintained and are available for purchase from Mosby until inventory is depleted. Please write to Mosby, Subscription Customer Service, 6277 Sea Harbor Dr, Orlando, FL 32877, or call $800-654-2452$ or $407-345-4000$ for information on availability of particular issues and prices. 